\section{Un nouveau type de traitement médical pourrait mener à la régression de la sténose valvulaire aortique}

David Busseuil, Eric Rhéaume, Jean-Claude Tardif
Centre de recherche, Institut de Cardiologie de Montréal, 5000 , rue Bélanger, Montréal (Québec), HIT 1 C8 Canada. dbusseuil@yahoo.com eric.rheaume@icm-mhi.org jean-claude.tardif@icm-mhi.org

inverse de cholestérol [6]. Puisque les HDL ont des propriétés anti-inflammatoires et favorisent le transport inverse du cholestérol, nous avons émis l'hypothèse qu'une thérapie fondée sur les HDL pourrait également entraîner la régression de la sténose valvulaire aortique.

\section{La sténose valvulaire aortique} calcifiée peut régresser

Le travail récent de notre laboratoire a porté sur la capacité d'un peptide mimétique de l'ApoA-I de provoquer une régression de la sténose valvulaire aortique calcifiée [7]. Nous avons utilisé un modèle de lapin précédemment décrit [8] dans lequel une diminution significative de la surface d'ouverture de la valve aortique est couplée à la présence de calcifications (obtenue par un régime enrichi en cholestérol $0,5 \%$ + vitamine $D_{2}$ dans l'eau de boisson), ce qui permet de reproduire les conditions observées en clinique. Dès 
7 jours après le début du traitement avec le peptide mimétique de l'ApoA-I, nous avons observé par échocardiographie une augmentation de la surface d'ouverture de la valve aortique, pour atteindre une amélioration maximale à 14 jours. La surface d'ouverture de la valve aortique à la fin des infusions du peptide mimétique de l'ApoAI $\left(23,8 \pm 3,1 \mathrm{~mm}^{2}\right)$ est presque revenue à sa valeur normale d'avant le début du régime hypercholestérolémique pour induire la sténose aortique $\left(24,7 \pm 3,9 \mathrm{~mm}^{2}\right)$. L'épaisseur de la valve aortique a été réduite de $21 \%$ après seulement 14 jours de traitement avec le peptide $(0,094 \pm 0,034 \mathrm{~cm}$ au début du traitement vs $0,075 \pm 0,033 \mathrm{~cm}$ à la fin du traitement) tandis qu'elle est restée inchangée chez les témoins ( $P$ $=0,0006$ ). L'analyse histologique des coupes de valves aortiques a montré que les infusions du peptide mimétique de l'ApoAI ont entraîné une réduction de la surface de lésion autour de la base du feuillet valvulaire (Figure 1).

Une telle réduction de la quantité des calcifications valvulaires est significative. Nous suggérons donc que ce traitement pourrait

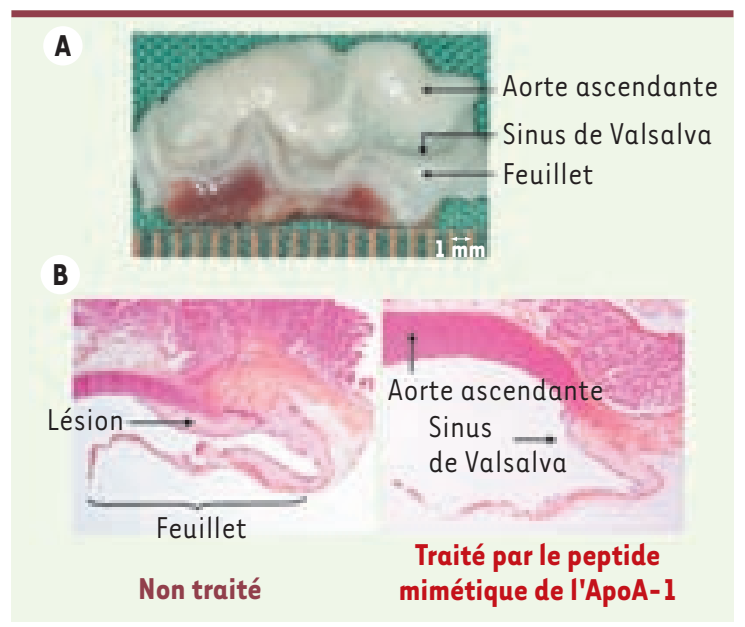

Figure 1. Valve aortique de lapin après régime hypercholestérolémique. A. On observe des dépôts lipidiques (relief blanchâtre) importants dans l'aorte ascendante, les sinus de Valsalva et les feuillets. Le tissu rouge situé sous les feuillets correspond au ventricule gauche. B. Sections longitudinales colorées avec haemaloxylin-phloxin-safran de valves aortiques de lapins. Gauche: lapin non traité. Droite : lapin traité par un peptide mimétique de I'ApoA-I. La surface de lésion (tissu rose pâle) était moins importante dans la valve aortique des animaux traités. être éventuellement appliqué en clinique étant donné que la sténose aortique calcifiée des personnes âgées est la forme la plus répandue dans les pays développés.

\section{Exploration du mécanisme d'action du peptide mimétique de l'ApoA-I}

Le peptide mimétique de l'ApoA-I complexé avec des phospholipides utilisé dans notre étude a montré sa capacité à mobiliser le cholestérol dans des études précliniques et est également capable d'élever les taux circulants de HDL-cholestérol chez les patients [9]. Dans notre étude, les taux de cholestérol total circulant élevés chez les lapins traités avec le peptide $(17,57 \pm 10,32 \mathrm{mM}$, observés après 2 semaines de traitement) indiquent probablement une augmentation de la mobilisation du cholestérol tissulaire. Nous avons également montré après coloration avec le filipin une diminution de l'intensité de la fluorescence du cholestérol libre présent dans la surface luminale des lésions valvulaires, qui pourrait être le résultat d'un plus grand flux de cholestérol aux lipoprotéines circulantes induit par le peptide. Compte tenu du fait que les taux plasmatiques de cholestérol étaient très élevés tout au long de l'étude $(20,46 \pm 3,52$ et $20,13 \pm 5,18 \mathrm{mM}$ au début du traitement, $13,78 \pm 6,24$ et $17,57 \pm 10,32 \mathrm{mM}$ à la fin du traitement, respectivement dans les groupes témoin et traité, $p=0,470$ ), nous n'avons pas observé une présence moindre de macrophages, mais ce phénomène n'implique pas nécessairement que cet ApoA-I mimétique ne possède pas de propriétés anti-inflammatoires intrinsèques. Une thérapie plus prolongée aurait pour effet possible d'entraîner une diminution de la surface occupée par les macrophages dans ce contexte.

\section{En conclusion}

Notre étude démontre que l'administration d'un peptide mimétique de l'ApoA-I entraîne la régression de la sténose valvulaire aortique expérimentale, ainsi qu'une diminution des calcifications. Ces résultats positifs justifient la nécessité de tester ce type de traitement chez les patients souffrant de cette pathologie. Si elle est obtenue de manière sécuritaire, la régression de la sténose aortique pourrait transformer l'approche clinique de cette pathologie, notamment en évitant une intervention chirurgicale lourde pour le patient. $\diamond$

A new type of medical treatment

could lead to regression

of aortic valve stenosis

\section{CONFLIT D'INTÉRÊT}

Un brevet provisoire a été soumis concernant les résultats de cet article par l'Institut de Cardiologie de Montréal et Dr Tardif est cité comme auteur.

\section{RÉFÉRENCES}

1. Freeman RV, Otto CM. Spectrum of calcific aortic valve disease: pathogenesis, disease progression, and treatment strategies. Circulation 2005 ; 111 : 3316-26.

2. Carabello BA. Aortic stenosis. N Engl J Med 2002 ; $346: 677-82$.

3. Nissen SE, Tsunoda T, Tuzcu EM, et al. Effect of recombinant ApoA-I Milano on coronary atherosclerosis in patients with acute coronary syndromes: a randomized controlled trial. JAMA 2003; $290: 2292-300$.

4. Tardif JC, Grégoire J, L'Allier PL, et al. Effects of reconstituted high-density lipoprotein infusions on coronary atherosclerosis: a randomized controlled trial. JAMA 2007 ; 297 : 1675-82.

5. Shah PK, Yano J, Reyes 0, et al. High-dose recombinant apolipoprotein A-I (milano) mobilizes tissue cholesterol and rapidly reduces plaque lipid and macrophage content in apolipoprotein e-deficient mice. Potential implications for acute plaque stabilization. Circulation 2001 ; 103 : 3047-50.

6. Meyers CD, Kashyap ML. Pharmacologic augmentation of high-density lipoproteins: mechanisms of currently available and emerging therapies. Curr Opin Cardiol $2005 ; 20: 307-12$.

7. Busseuil D, Shi Y, Mecteau M, et al. Regression of aortic valve stenosis by ApoA-I mimetic peptide infusions in rabbits. BrJ Pharmacol 2008 ; 154 : 765-73.

8. Drolet MC, Arsenault M, Couet J. Experimental aortic valve stenosis in rabbits. J Am Coll Cardiol 2003 ; $41: 1211-7$.

9. Khan M, Lalwani ND, Drake SL, et al. Single-dose intravenous infusion of ETC-642, a 22-Mer ApoA-I analogue and phospholipids complex, elevates HDL-C in atherosclerosis patients. Circulation 2003; 108 (suppl IV) : 563-4. 